\title{
The electric current approach in the solar-terrestrial relationship
}

\author{
Syun-Ichi Akasofu \\ International Arctic Research Center, University of Alaska Fairbanks, Fairbanks, AK 99775, USA \\ Correspondence to: Syun-Ichi Akasofu (sakasofu@iarc.uaf.edu)
}

Received: 27 March 2017 - Revised: 12 April 2017 - Accepted: 3 May 2017 - Published: 21 August 2017

\begin{abstract}
The sequence of phenomena consisting of solar flares, coronal mass ejections (CMEs), auroral substorms, and geomagnetic storms is mostly a manifestation of electromagnetic energy dissipation. Thus, first of all, it is natural to consider each of them in terms of a sequence of power supply (dynamo), power transmission (electric currents/circuits), and dissipation (mostly observed phenomena), i.e., as an input-output process and the electric current line approach. Secondly, extending this concept, it is attempted in this paper to consider the whole solar-terrestrial relationship in terms of electric currents. This approach enables us to follow through not only the sequence in solar flares, auroral substorms, and geomagnetic storms but also to connect all phenomena naturally as a continuous flow of magnetic energy $\left(V\left[B^{2} / 8 \pi\right]\right)$ from the sun across the magnetopause. This consideration gives some insight into all the processes involved equally well compared with the magnetic field line approach, which has been adopted almost exclusively in the past.
\end{abstract}

Keywords. Ionosphere (electric fields and currents)

\section{Introduction}

In as early as 1967, Alfvén (1967) noted the following: "In some application we can illustrate essential properties of the electromagnetic state of space either by depicting the magnetic field lines or by depicting the electric current lines. Almost always the first picture is used exclusively. It is important to note that in many cases the physical basis of the phenomena is better understood if the discussion is centered on the picture of the current lines". Alfvén (1977) also emphasized the following: "Hence in order to understand the properties of a current-carrying plasma, we must take account of the properties of the whole circuit in which the current flows". His point is that it is necessary to consider the whole sequence (dynamo, transmission, and dissipation) as an input-output process.

Indeed, the current line approach can follow quantitatively the flow of the power/energy (physical quantities) as a continuous process through the whole sun-earth system and thus can link the resulting phenomena. For this reason, it is worthwhile to consider the sequence of solar flares, coronal mass ejections, auroral substorms, and geomagnetic storms in terms of the electric current approach.

\section{Power supply}

In all the phenomena we consider in this paper, the first important subject is the power supply produced by plasma flows in a magnetic field.

The dynamo power $P$ is given by the Poynting flux:

$P=\int E \times B \cdot \mathrm{d} S=V\left(B^{2} / 8 \pi\right) S$,

where $V$ and $B$ denote the speed of plasma flows and the magnetic field intensity in the photosphere; $S$ is the cross section which depends on the phenomena we deal with (cf. Akasofu, 1977, chap. 1, Sect. 1.4). The attempt in this paper is to follow the flow of the dynamo power/energy $V\left(B^{2} / 8 \pi\right)$ from solar flares/coronal mass ejections (CMEs) to auroral substorms/geomagnetic storms on the basis of the current line approach.

\section{Solar flares}

In terms of the current line approach, solar flare processes can be described by the following terms: photospheric dynamo, the magnetic arcade current system (the $H \alpha$ emission), and the loop (prominence) current system (explosive prominences and CMEs). 
This approach may be contrasted with the present magnetic field line approach, which involves the following: magnetic reconnection (converting magnetic energy) and CMEs and downward plasma flows (causing flare phenomena, like the $H \alpha$ emission).

In considering solar flares in terms of the current line approach, it is important to recognize that there are two circuits: the magnetic arcade circuit and the loop (prominence) circuit.

The first one is the magnetic arcade current system that is directly driven by the photospheric dynamo, as described in the following section; its dissipation is manifested as the $H \alpha$ (two-ribbon) emission along the feet of the arcade. In this case, the dynamo power is dissipated directly at the feet of the magnetic arcade, so that the magnetic energy conversion is not needed.

The second one is a loop current system along a filament (prominence) above and along the arcade, in which the magnetic energy is accumulated and then released when the loop current is reduced (Fig. 3). This current is crucial in generating and launching CMEs and eventually causing substorms/storms.

\subsection{Need for photospheric dynamo as the power supply}

There have been intensive studies on solar flares in the past; some of the recent studies include those by Webb et al. (2000), Fletcher and Hudson (2008), Filippov and Koutchmy (2008), Zao (2009), Wang and Liu (2010), Milligan et al. (2010), Schuck (2010), Steward et al. (2011), Su et al. (2013), Kerr and Fletcher (2014), Aschwanden et al. (2014, 2015), Aschwanden (2015), Fisher et al. (2015), and Kazachenko et al. (2015), in addition to reviews by Zharkova et al. (2011), Hudson (2011), and Shibata and Magara (2011).

Most recent studies of solar flares are based on magnetic reconnection at the outset as the conversion process of magnetic energy or the "merging" of antiparallel magnetic field lines, and they then consider its consequences. They have not considered the power supply, assuming there is enough magnetic energy around sunspots (cf. Shibata and Magara, 2011). Thus, in their approach, the concept of power supply by a dynamo is lacking.

In spite of such a great emphasis and of numerous theoretical and observational studies on magnetic reconnection, there has been a puzzling omission of estimating accurately the magnetic field intensity and the amount of available magnetic energy at the location where magnetic reconnection is supposed to occur. The only exceptions are an estimate by Priest (1981, p. 139), who mentioned the field intensity to be $B=500 \mathrm{G}$ without mentioning the location, and Shibata and Magara (2011), who mentioned $B=1000 \mathrm{G}$ "in a typical sunspot". Thus, the first question in this approach is where magnetic reconnection is supposed to occur and whether or not there is enough energy there.

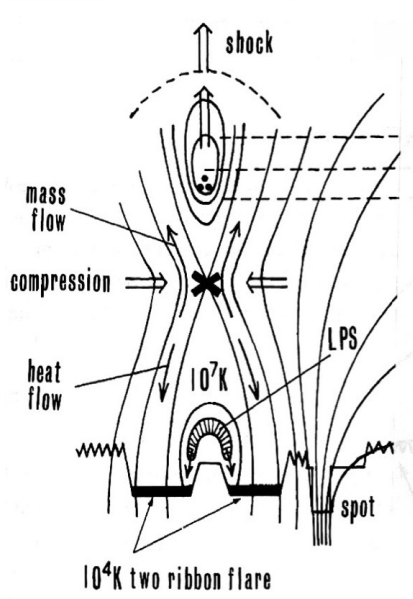

(a)

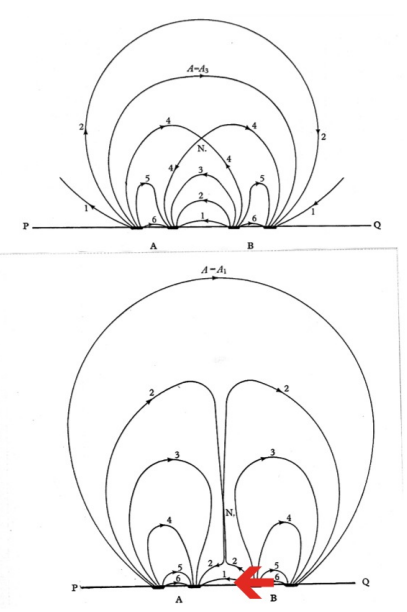

(b)
Figure 1. (a) Magnetic reconnection model in the corona above a magnetic arcade (Hirayama, 1974). (b) Two approaching sunspot pairs, forming an antiparallel magnetic field configuration (Sweet, 1958); a red arrow is added to show the motion of a sunspot pair.

One of the generally accepted models assumes magnetic reconnection above the magnetic arcade (Fig. 1a). Unfortunately, there have so far been neither measurements (not possible at present) of the magnetic field intensity in the corona nor estimates of it; most coronal magnetic field models assume the current-free condition, so that there is no expendable magnetic energy under such a condition. Although the field above the magnetic arcade is not current free, it may not be very different from the situation similar to the transition region from the main body of the magnetosphere to the tail region (the magnetotail). Thus, let us try to roughly estimate the field intensity where magnetic reconnection is supposed to occur above the arcade. The width of the arcade is likely to be the distance of two-ribbon flares, namely $2.5 \times 10^{4} \mathrm{~km}$ (corresponding to the diameter of the earth). Assuming field intensity of as large as $100 \mathrm{G}$ on both sides of two unipolar regions (corresponding to $B$ in the polar region of the earth), the field intensity at the top of the arcade (corresponding to the earth's equator) will be about $100 \mathrm{G} / 2=50 \mathrm{G}$. Aschwanden (2004, p. 601) suggested that magnetic reconnection occurs above $4.4 \times 10^{4} \mathrm{~km}$. Assuming the height to be $5 \times 10^{4} \mathrm{~km}$, it is twice the height of the arcade, where an estimated $B$ will be $50 \mathrm{G} / 2^{3}=6.25 \mathrm{G}$ at that distance. Since the magnetic field there is not current free, let us assume $B$ is $10 \mathrm{G}$ there; thus, the corresponding minimum volume needed for the weakest flares $\left(10^{30} \mathrm{ergs} /\left[B^{2} / 8 \pi\right]\right)$ is a sphere of radius about $6 \times 10^{4} \mathrm{~km}$, which seems to be too large even for the weakest flares, compared with the height of the arcade. Thus, it is likely that there is not enough magnetic energy for flares at the height of $5 \times 10^{4} \mathrm{~km}$. 


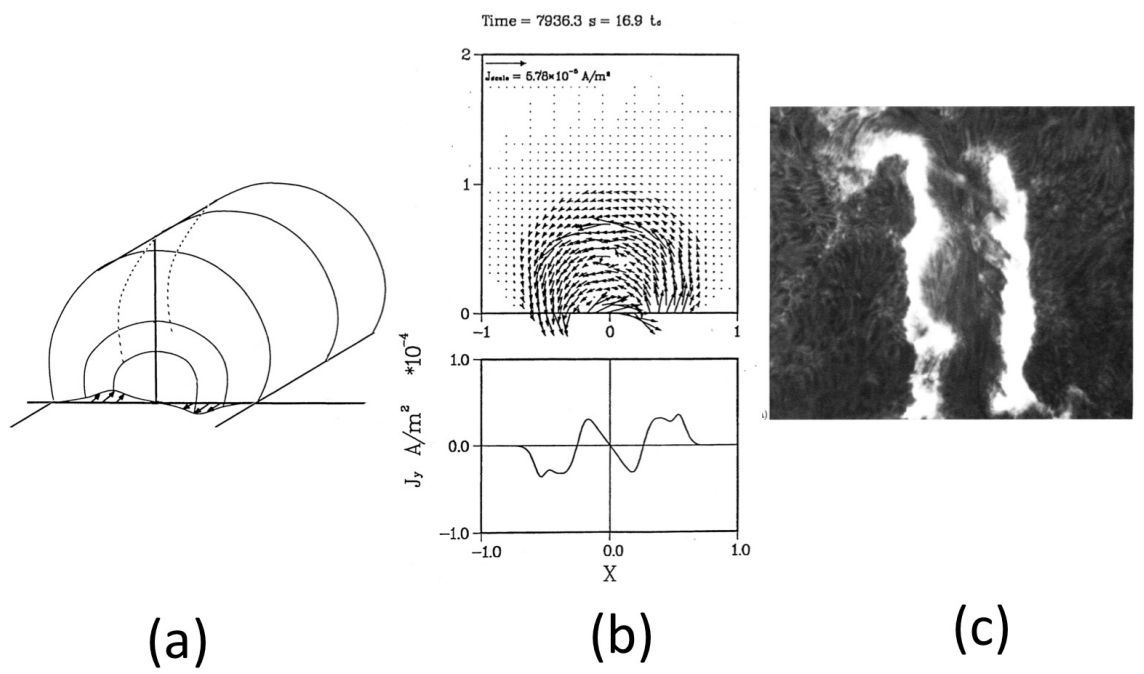

Figure 2. (a) The magnetic arcade with an antiparallel plasma flow in the photosphere. (b) The current distribution in the magnetic arcade (Choe and Lee, 1996a, b). (c) Spotless two-ribbon flare (courtesy of H. Zirin, 1985).

The second problem is the absence of the concept of power supply for the historical reason mentioned earlier in the present solar flare study. It can be shown in the following that the power supply is needed not only for the formation of an antiparallel magnetic configuration but also for maintaining flare activities.

Actually, the need for a dynamo in producing an antiparallel configuration was already implicitly mentioned in the earliest study of magnetic reconnection for flares. Sweet (1958) proposed that two approaching (or colliding) sunspot pairs produce an antiparallel magnetic configuration, allowing for magnetic reconnection to release the magnetic energy for flares (Fig. 1b). It is important to recognize that his hypothetical motion of sunspot pairs implicitly expresses the need for a photospheric-dynamo process, producing an antiparallel magnetic configuration and maintaining the power/energy supply.

In order to prove the above statement, let us consider first Sweet's conceptual case on the basis of commonly observed values of these parameters $P, V, B, S$, and the dissipation rate $\delta$. Here, we use the minimum total energy $W=10^{30} \mathrm{ergs}$ of solar flares, so that the minimum power $P$ needed is estimated to be about $2.8 \times 0^{26} \mathrm{erg} \mathrm{s}^{-1}\left(=10^{30} \mathrm{erg} \mathrm{h}^{-1}\right.$ ), assuming that flares typically last for $1 \mathrm{~h}$. Large $H \alpha$ flares are associated with a dissipation rate of about $10^{26} \mathrm{erg} \mathrm{s}^{-1}$ (Svestka, 1976, p. 13). Let us first consider that a sunspot pair with an area of rectangular size $L=5 \times 10^{4} \mathrm{~km}$ approaches another sunspot pair of intensity $B=100 \mathrm{G}$, with a speed $V$ of $1 \mathrm{~km} \mathrm{~s}^{-1}$; for simplicity, $V$ is assumed to be perpendicular to $B$.

These numbers are quite common and reasonable in the vicinity of sunspot groups and can provide power $P=2.0 \times$ $10^{26} \mathrm{erg} \mathrm{s}^{-1}$ (the minimum power needed for solar flares), assuming dynamo depth of $500 \mathrm{~km}$. There have been sev- eral measurements of plasma flows beneath active sunspot areas $\left(0.1-18 \mathrm{Mm} ; \mathrm{Mm}=10^{3} \mathrm{~km}\right)$ by Kosovichev and $\mathrm{Du}-$ vall Jr. (2010); although there have been no such observations specifically associated with solar flares, the speed of plasma is observed to be up to $50 \mathrm{~m} \mathrm{~s}^{-1}-1 \mathrm{~km} \mathrm{~s}^{-1}$ under active sunspots.

The purpose of the above estimates is simply to demonstrate the need for a dynamo as the power supply even for magnetic reconnection. Therefore, although in this paper magnetic reconnection is not considered as the process of magnetic energy conversion, it is clear that a photosphericdynamo process is needed to produce even the desired antiparallel magnetic configuration for magnetic reconnection and to maintain the power supply.

\subsection{The photospheric dynamo and its circuits}

\subsubsection{The dynamo under a magnetic arcade and its circuit}

Choe and Lee (1996a, b) developed a photospheric-dynamo model of solar flares, including active prominences. Its basis is a magnetic arcade which is formed along the boundary (the neutral line) between two unipolar regions, where no sunspot pairs or sunspot groups are present. This model was originally developed by Hirayama (1974) as a magnetic reconnection model (Fig. 1a), so it did not consider the power supply, namely photospheric plasma flows in the magnetic arcade; a photospheric dynamo is needed to produce the antiparallel field to begin with and supplies the power. Figure $2 \mathrm{a}$ shows the geometry associated with the dynamo considered by Choe and Lee (1996a, b). Their model assumes an antiparallel plasma flow $\left(V=2 \mathrm{~km} \mathrm{~s}^{-1}\right)$ along the centerline of the arcade, which is the boundary of two unipolar regions of 
opposite polarity, and the magnetic field intensity $B=12 \mathrm{G}$ $(=6 \mathrm{G}+6 \mathrm{G})$. These values are well within the observational constraints along the neutral line between two unipolar magnetic regions.

Choe and Lee's (1996a, b) computer simulation is extended here. An additional calculation shows that the fieldaligned currents flow along the arcade magnetic field lines generated by this dynamo process. Thus, the arcade magnetic field lines are the circuit which transmits the dynamo power to the chromosphere. The current flows along the feet of the magnetic arcade for a two-ribbon flare (one of the main dissipations). There is no need for magnetic reconnection.

\subsubsection{Dissipation}

The intensity of the field-aligned current is about $10^{-4} \mathrm{~A} \mathrm{~m}^{-2}$, corresponding to an electron flux of $10^{14} \mathrm{~m}^{-2} \mathrm{~s}^{-1}$. The results are shown in Fig. 2b. For the area of typical two ribbons of $H \alpha$ emission $\left(2 \times 2 \times 10^{5} \mathrm{~km} \times 2.5 \times 10^{4} \mathrm{~km}\right)$, the total current and the energy carried by the electrons are, respectively, about $10^{12} \mathrm{~A}$ and $10^{25} \mathrm{erg} \mathrm{s}^{-1}$ (as demonstrated by auroral electrons, the current-carrying electrons are accelerated to $1000 \mathrm{keV}$ along the magnetic field lines of the arcade, in order to penetrate into the chromosphere). As mentioned earlier, large $H \alpha$ flares are associated with an energy of about $10^{26} \mathrm{erg} \mathrm{s}^{-1}$ (Svestka, 1976, p. 13). Thus, the photospheric-dynamo model by Choe and Lee (1996a, b) can provide the current/circuit and the power dissipation (the two-ribbon $H \alpha$ emission) for the simplest and weakest flares, that is at least for spotless flares (Fig. 2c). It is expected that the intensity of the $H \alpha$ emission follows closely the power of the photospheric dynamo as a function of time (the so-called "explosive" nature of flaring is due in part to a speeded-up time-lapse video).

Therefore, the study by Choe and Lee (1996a, b) suggests that the photospheric dynamo alone can directly produce the two-ribbon $H \alpha$ emission at the feet of the magnetic arcade, one of the major dissipation processes, and thus that the power is carried by the current along the arcade magnetic field lines to the chromosphere. In this model (spotless flare), neither sunspots nor magnetic reconnection is involved in producing the $H \alpha$ emission (one of the major dissipation processes). In this case, $P(t)=\delta(t)$.

Intense flares tend to occur among an active sunspot group. In particular, the two-ribbon emission tends to occur along a neutral line in an active sunspot group. It is expected that both $V$ and $B$ are large within an active sunspot group, so that the power is also expected to be greater. In the case of Choe and Lee's (1996a, b) model, $B$ is $12 \mathrm{G}$, but it is expected to be much larger, say $100 \mathrm{G}$ or more, along the neutral line (along which two-ribbon flares tend to occur) in an active sunspot group, so that the power is expected to become $10^{32}$ ergs or more. Therefore, their dynamo model discussed above should also be applicable to much more intense flares.

\subsection{Loop current}

\subsubsection{Circuit}

One of the most interesting features of flare phenomena is active prominences, which require processes other than the $H \alpha$ emission. Active prominences are often observed as dark filaments along the top of the magnetic arcade and expand/explode during flares (Fig. 3a). Electric currents flow in a filament (prominence) structure (Bothmer and Schwenn, 1994), forming a current loop (Fig. 3b). Choe and Lee (1996a, b) also showed the formation of such a current above and along the magnetic arcade.

\subsubsection{Dissipation}

A sudden reduction in the loop current is expected to release its magnetic energy (Alfvén and Carlqvist, 1967). Let us assume that the magnetic energy is accumulated when the loop current is building up.

The magnetic energy $W$ in a loop current is given by

$W=(1 / 2) J^{2} L$,

where $L$ denotes the inductance in the loop circuit. Chen and Krall (2003) estimated the intensity of their loop current to be $10^{11} \mathrm{~A}$ in their theory of CMEs. For medium-intensity flares ( $W=10^{32} \mathrm{ergs}$ ), $L$ is estimated to be $2000 \mathrm{H}$.

The conversion of magnetic energy $W$ into the energy of prominence eruption can be considered to be a process of a rapid current reduction and the resulting release of energy. The reduction process is likely to be a current instability rather than magnetic reconnection. Indeed, instabilities of filaments during flares have been observed by Moore et al. (2001), Rust and LaBonte (2005), and many others. It is interesting to note that an expanding loop appears to have untwisting motions, suggesting a sudden reduction in the loop current (Kurokawa et al., 1987).

\subsubsection{Launching coronal mass ejection}

The released magnetic energy is expected to cause CMEs. Chen and Krall (2003) showed that the Lorentz force $(J \times B)$ is responsible for ejecting CMEs (overcoming the solar gravity) rather than that they are the results of a thermal expansion (pressure gradient) or magnetic reconnection; thus, the loop current in prominences is vital in launching CMEs from the point of view of the current line approach. As shown in the next section, it is difficult to explain a helical magnetic structure of CMEs without considering such an expanding current loop.

This is an example to show that the current line approach suggests that there are two current circuits and can also suggest a requirement of the initial condition in launching CMEs, when CMEs have a helical magnetic structure (Sect. 3), which is crucial in predicting the intensity of auroral substorms/geomagnetic storms. Thus, when CMEs have a 


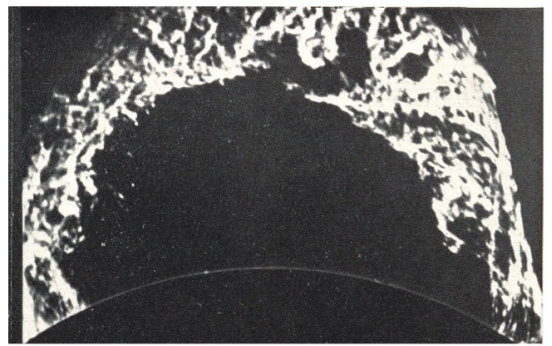

(a)

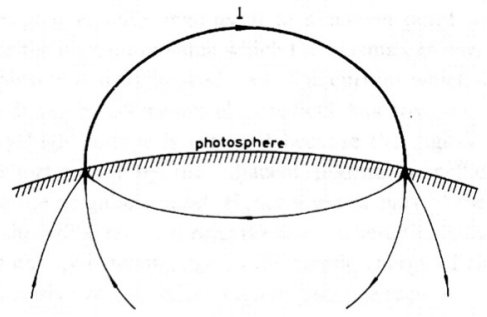

(b)

Figure 3. (a) An example of expanding prominences (courtesy of W. O. Roberts, 1970). (b) A current loop for a prominence (Alfvén and Carlqvist, 1967).
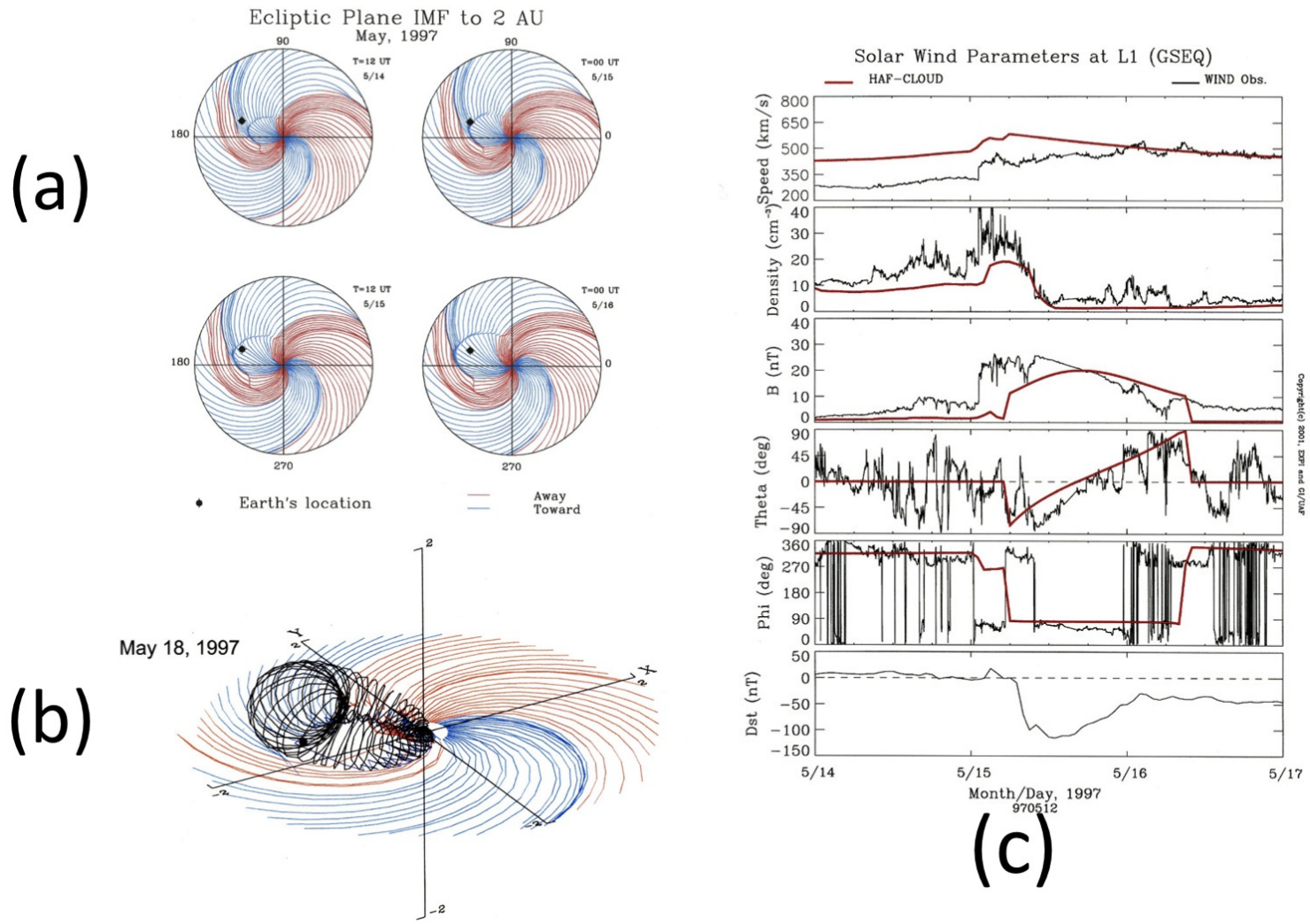

Figure 4. (a) The propagation of the shock wave associated with the 14-15 May 1997 geomagnetic storm (Fry et al., 2001); the location of the earth is indicated by a dot. (b) The reconstructed of the helical magnetic structure (Saito et al., 2007). (c) Comparison of results between the observation and the reconstructed helical structure (Saito et al., 2007; Akasofu, 2017).

clear helical magnetic structure, one of the initial conditions should specifically include the expanding loop current rather than a thermal expansion or magnetic reconnection alone.

\section{CMEs: current loop}

Some CMEs have a helical magnetic structure (cf. Burlaga et al., 1981). Furthermore, Gosling et al. (1986) showed that some of magnetic loops have their feet on the photosphere. Thus, at least some CMEs have current loops. However, magentohydrodynamics (MHD) simulations of CMEs have so far not clearly showed the observed helical magnetic configuration (cf. Odstrci et al., 2004; Wu et al., 2007; Lugaz et al., 2009).

Thus, by assuming currents of $10^{9} \mathrm{~A}$ along the loop, an attempt was made to reconstruct the helical magnetic struc- 
ture (Saito et al., 2007). An example is shown in Fig. 4 (1415 May 1997). This reconstruction is made after the event. Nevertheless, the fact that we can reconstruct crudely some features of the magnetic structure of CMEs, as well as the number density and the speed, by assuming a loop current with the Hakamada-Akasofu-Fry (HAF) model (Fry et al., 2001), suggests that this approach may help in guiding MHD simulation models. Indeed, Bothmer and Schwenn (1994) showed that some prominences have a helical magnetic configuration.

It is suggested that both methods should work together, instead of considering the MHD simulation approach alone. In the previous section, we emphasized that the initial condition in launching CMEs of a helical structure should have a current loop associated with active prominences (Chen and Krall, 2003).

As will be shown in the next section, the magnetic structure of CMEs is crucial in predicting the intensity and its time variations in geomagnetic storms, which are a vital part of space weather science. The polar angle $\theta$ of the interplanetary magnetic field (IMF) plays a crucial role in determining the dynamo process and the power generated by the interaction between the solar wind and the magnetosphere (Sect. 4.1); $V$ and $B$ alone are insufficient. Even if $V$ and $B$ can be very large, there will be no power if $\theta=0$. This is the reason why intense flares at the center of the solar disk often fail to cause major geomagnetic storms, Thus, it is essential to predict the magnetic configuration for the intensity of geomagnetic storms and auroral substorms, not just the intensity of flares and plasma flow speed.

\section{Auroral substorms}

In terms of the current line approach, magnetospheric processes associated with auroral substorms can be described as a combination of the following: the Chapman-Ferraro current (see Sect. 5.1), the solar wind-magnetosphere dynamo, the directly driven (DD) current system, the unloading component (UL) current, and the ring current as a result of loading/unloading of magnetic energy in the main body of the magnetosphere.

This approach may be contrasted with the magnetic field line approach, which involves the following: dayside reconnection, flux transfer, magnetic reconnection in the magnetotail, and the resulting plasma flows and their consequences (in producing the substorm current system and the ring current).

\subsection{Solar wind-magnetosphere dynamo}

The solar wind-magnetosphere dynamo power has been empirically expressed by Perrault and Akasofu (1978):

$\varepsilon=V B^{2} \sin ^{4}(\theta / 2) l^{2}$, where $V$ and $B$ denote the solar wind speed and the magnitude of the IMF and $\theta$ is the polar angle of the IMF; $l^{2}$ is the dimensional factor, and $l$ is taken to be $15 R_{\mathrm{E}}$ (earth's radius). Rewriting the above equation in terms of the Poynting flux $V\left(B^{2} / 8 \pi\right) \sin ^{4}(\theta / 2) l^{2}, l$ become $5 R_{\mathrm{E}}$, where $S=\sin ^{4}(\theta / 2) l^{2}$; for this theoretical derivation, see Akasofu (1977, chap. 1.4).

There are two important implications in the above expression of the power. From the point of view of the energy flow from the sun, the above expression of the power indicates that the magnetic energy $\left(B^{2} / 8 \pi\right)$ is carried out by the solar wind with the speed $V$ into the heliosphere and that the magnetosphere has a sort of "cross section" or "filter" $\left[\sin ^{4}(\theta / 2) l^{2}\right]$ to receive it. At the earth's distance, for $B=5 \mathrm{nT}$ and $V=500 \mathrm{~km}$, the magnetic energy flux is $5 \times 10^{-3} \mathrm{erg} \mathrm{cm}^{-2} \mathrm{~s}^{-1}$, which may be compared with the particle flux $7.0 \times 10^{-2} \mathrm{erg} \mathrm{cm}^{-2} \mathrm{~s}^{-1}$ and the black-body radiation flux of $3.4 \times 10^{5} \mathrm{erg} \mathrm{cm}^{-2} \mathrm{~s}^{-1}$.

The second implication is that the magnetic energy carried by the solar wind (which is identical to the power of the solar wind-magnetosphere dynamo, the Poynting flux) flows across the magnetopause and flows in the direction perpendicular to the magnetic field lines, so that a significant amount of the power thus generated flows across the magnetopause toward a dipole-like field of the main body of the magnetosphere. This point is very important in considering where the magnetic energy is accumulated for the expansion phase

\subsection{Currents/circuits}

The substorm current has two components: the DD component and the UL; Akasofu (2013). The DD component is directly driven by the solar wind-magnetosphere dynamo of power $\varepsilon$, while the UL component is a result of the unloading of the loaded magnetic energy during an early epoch (the growth phase) in the main body of the magnetosphere (within a distance of $10 R_{\mathrm{E}}$ ). The current line approach succeeded in identifying the current system UL which is directly responsible for the expansion phase. Both current systems are illustrated in Fig. 5a and b. The DD component has been constructed by a large number of researchers, including Chapman (1935), Dungey (1961), and Axford and Hines (1961). The UL component was proposed by Bostrom (1964).

Figure 6 shows how the DD and UL components vary during substorms, together with $\varepsilon$. It can be seen that the DD component tends to follow $\varepsilon$, while the UL component is independent of $\varepsilon$ and impulsive. It is the UL component which is responsible for spectacular auroral displays during the expansion phase (particularly the poleward advance; Sect. 4.6). In a sense, DD corresponds to the magnetic arch current and UL to the loop current in solar flares. 


\section{DD component}

(a)

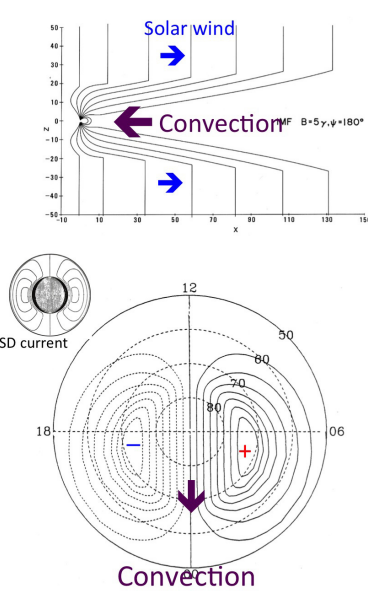

Ionosphere

(d) (b)

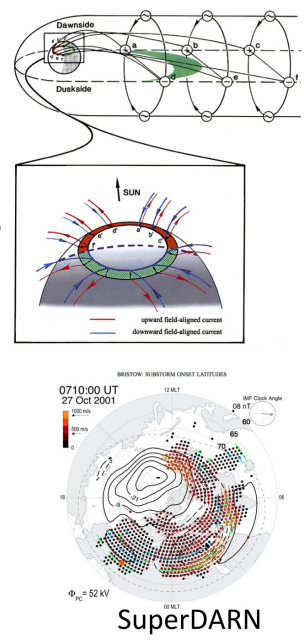

(e) (c)
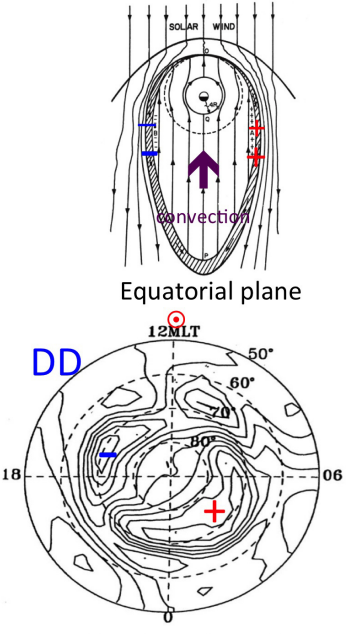

(f)

\section{UL component}

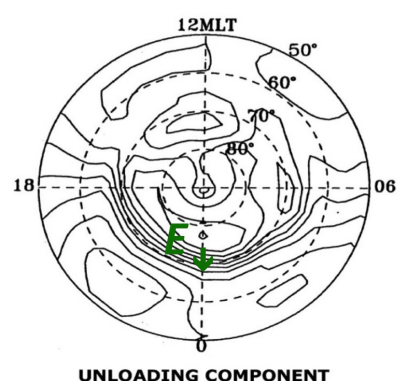

(g)

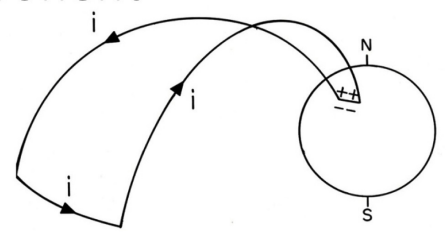

Azimuthal loop

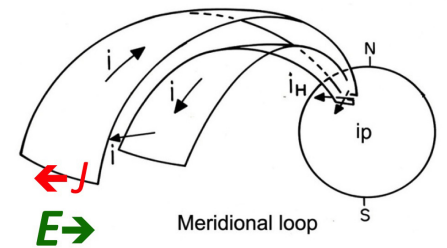

$E \bullet J<0$

(h)

Figure 5. The DD current: (a) the solar wind-magnetosphere interaction; (b) a schematic illustration of the circuit; (c) the resulting convection pattern of magnetospheric plasmas (Axford and Hines, 1961); (d) the expected convection pattern of ionospheric plasma, which is basically the equipotential pattern (Akasofu, 2017); (e) SuperDARN result (Bristow and Jensen, 2007); (f) the realistic ionospheric DD current (convection) derived from the magnetometer data (Akasofu, 2017). The UL current: (g) the UL component of the ionospheric current (Akasofu, 2017); (h) Bostrom's 3-D current system made up of azimuthal and meridional components (Akasofu, 2017).

\subsection{Dissipation}

\subsubsection{Direct dissipation}

The major dissipation associated with auroral substorms is the Joule heat production in the ionosphere. The amount of the Joule dissipation can tell us how much energy a single substorm consumes and thus also how much energy the magnetosphere can accumulate. The current line approach is so far the only way to provide this crucial quantity observation- ally. The Joule heat production is proportional to the intensity of the current in the ionosphere because it is proportional to $J^{2} / \sigma=(J / \sigma) J$, where $\sigma$ is the ionospheric conductivity (which is proportional to $J$ ). Figure 7 shows an example of the distribution of dissipation in the ionosphere during a substorm. By integrating the dissipation over the polar ionosphere, one can determine the global dissipation rate $\delta(t)$, which is about $3-5 \times 10^{18} \mathrm{erg} \mathrm{s}^{-1}$. Integrating $\delta(t)$ further during a substorm, one can obtain the total dissipation dur- 


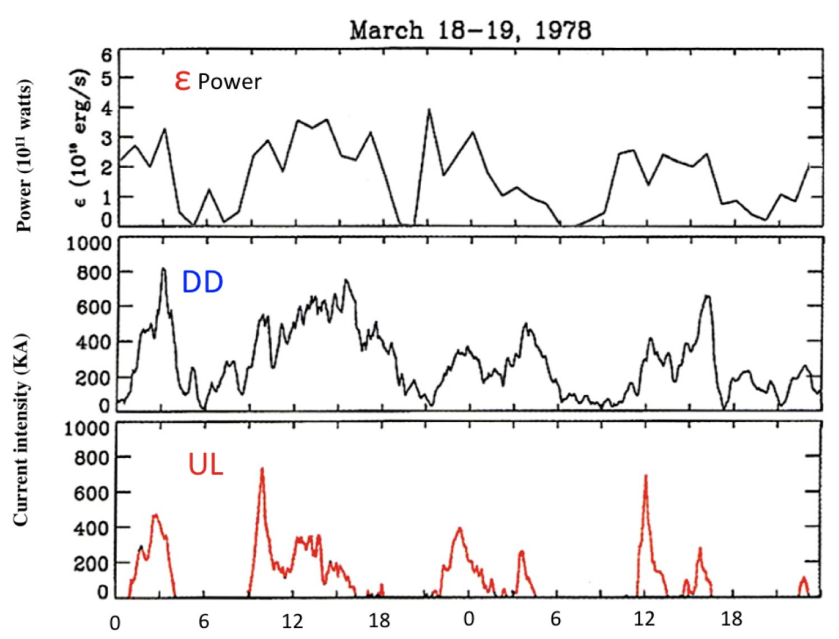

Figure 6. Time variations in the power $\varepsilon$, the DD current, and the UL current (Sun et al., 1998). Note that the DD tends to follow $\varepsilon$ but the UL is impulsive (Akasofu, 2013).

ing a substorm; it is on average $5 \times 10^{22} \mathrm{ergs}$ for mediumintensity substorms.

\subsubsection{Loading and unloading}

\section{Loading}

When the magnetic energy is accumulated in the main body of the magnetosphere, the magnetosphere is loaded and thus "inflated". Figure 8 shows a second-order calculation of the inflation centered at $6 R_{\mathrm{E}}$ in a symmetric ring current (Akasofu and Chapman, 1961; Akasofu et al., 1961). It can be seen that the magnetic field is pushed out from the region where the energy is accumulated. The accumulated magnetic energy is $2.9 \times 10^{21} \mathrm{ergs}$, and the $\beta$ value $\left(=\mathrm{nkT} /\left[B^{2} / 8 \pi\right]\right)$ becomes close to 1.0 at $6 R_{\mathrm{E}}$ in this case. The current density is $3 \times 10^{-18} \mathrm{~A} \mathrm{~cm}^{-2}$ at $6 R_{\mathrm{E}}$.

\section{Unloading and the magnetic energy conversion}

There is uncertainty about the limiting amount of magnetic energy that makes the magnetosphere unstable. Since $\beta$ becomes close to 1.0, the above amount of magnetic energy is not far from the maximum amount of energy which can be loaded or accumulated around $6 R_{\mathrm{E}} ; \beta=1.0$ may be a measure of such instabilities, because the magnetic field may not be able to control the dynamics of the plasma well, and thus the system will become unstable.

For these reasons, it is expected that the magnetosphere tries to stabilize itself, resulting in the unloading of the accumulated magnetic energy. It is suggested that the expansion phase of auroral substorms is a consequence of this process.

In order to illustrate the two roles of the magnetosphere, a tippy bucket has been considered to represent the UL component. The development of individual substorms is very com-

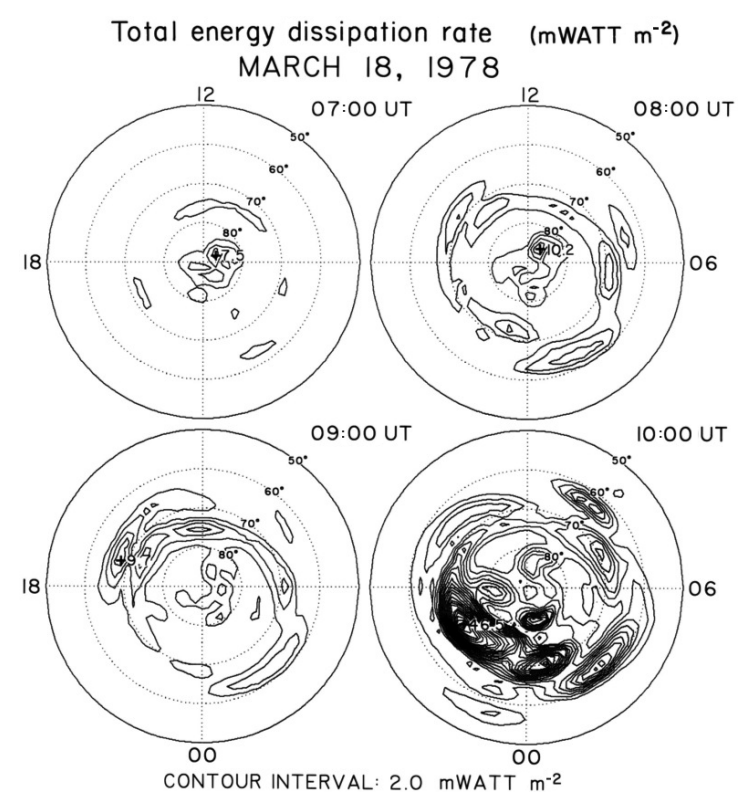

Figure 7. Joule heat production. The distribution of the Joule heat production in the ionosphere during a substorm, including a quiet period and the growth phase (prior to the expansion onset) (Akasofu, 2017).

plicated, because the input function $\varepsilon(t)$ is not the same, and the dissipation depends on various conditions of the ionosphere and the magnetosphere, so that several possibilities can be considered, depending on individual substorms (Zhou and Tsurutani, 2002, their Fig. 5). However, the basic case is very simple (Akasofu, 2015, his Fig. 9).

The magnetosphere is capable of accumulating more magnetic energy at closer distances (where $B^{2} / 8 \pi$ is higher) than at $6 R_{\mathrm{E}}$. It is suggested that the accumulated energy is located between 10 and $4 R_{\mathrm{E}}$, depending on the intensity of substorms $(\mathrm{AE}=100 \mathrm{nT}->2000 \mathrm{nT})$. Indeed, the onset latitude of the expansion phase can be as low as $48^{\circ}$ MLAT ( $L=2.4$ ) during major storms (although both the plasma sheet and the ring current shift closer to the earth during major storms, it is unlikely that the location of the accumulation is as close as $2.4 R_{\mathrm{E}}$ ). In this respect, it is interesting to know that the ratio of $\left(B^{2} / 8 \pi\right)$ at $4 R_{\mathrm{E}} / 10 R_{\mathrm{E}}$ is about 20 , corresponding to the substorm intensity ratio of $2000 \mathrm{nT} / 100 \mathrm{nT}$. The processes related to the power $\varepsilon$ and thus the DD current process, namely an enhanced convection, may be responsible for shifting the accumulation location.

The conversion or unloading process of the accumulated magnetic energy is likely to be different in the magnetotail and in the main body of the magnetosphere. Since magnetic reconnection is rare within $10 R_{\mathrm{E}}$ (Ge and Russell, 2006), a new process of conversion may be needed. In explaining the expansion phase, the most important requirement is that the converted magnetic energy must produce the UL current system (Fig. 5h) and thus an earthward electric field; the avail- 

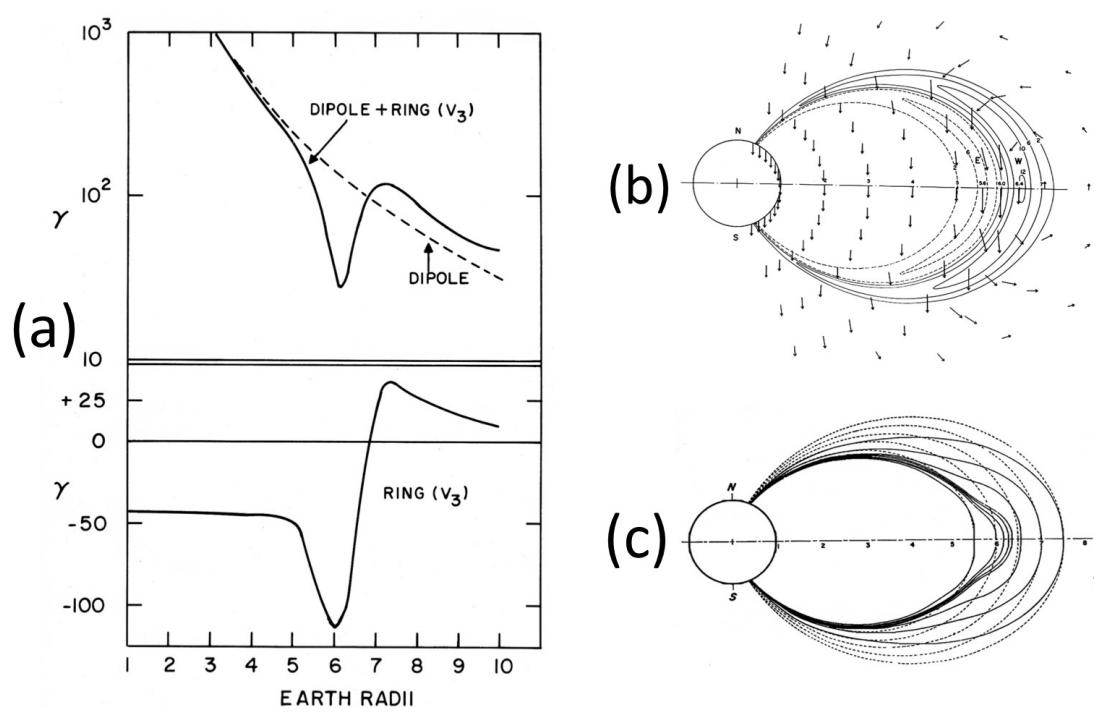

Figure 8. Changes in the earth's magnetic configuration when magnetic energy is accumulated centered around $6 R_{\mathrm{E}}$ (red dot); (a) the earth's field change and (b) the distribution of the magnetic field produced by the model ring current. (c) The distortion (inflation) of the magnetic field lines (Akasofu et al., 1961; Akasofu, 2017).

ability of energy is not enough. The current line approach can provide such a crucial requirement, which is also needed as the requirement for the magnetic field line approach; the plasma flow from the magnetotail must produce an earthward electric field.

Lui and Kamide (2003) and Akasofu (2013, 2015) suggested that electrons and protons are separated during the deflation and produce the earthward electric field needed (Fig. 9).

When the magnetic energy is accumulated, the magnetosphere is inflated. Thus, the unloading (or releasing) of the magnetic energy causes deflation. Because of the deflation $\left(\partial B_{z}>0\right)$ in the inflated location, an earthward electric field of $5 \mathrm{mV} \mathrm{m}^{-1}\left[=\left(-\partial B_{z} / \partial t \times\right.\right.$ $\left.\left.\int \partial y\right)=+50 \mathrm{nT} / 20 \mathrm{~min} \times 20 R_{\mathrm{E}}\right]$ can be produced, which can drive the UL current system. Depending on the values of the parameters in the above, the electric field could be as high as $50 \mathrm{mV} \mathrm{m}^{-1}$. The UL current system is mainly responsible for various expansion phase phenomena, including visible auroras and the auroral electrojet in the ionosphere. This process will continue as long as the magnetosphere is being deflated or until the accumulated energy is spent. The deflation time is less than $1.5 \mathrm{~h}\left(5 \times 10^{22} \mathrm{ergs} / 3-5 \times 10^{18} \mathrm{erg} \mathrm{s}^{-1}\right)$, which is similar to the period of the growth phase. Note that this short life similar to the period of the growth phase is also a requirement for the magnetic energy conversion; the duration of the expansion phase (which is similar to the duration of the growth phase) is also a requirement in explaining the expansion phase.

It may be noted that Lyons et al. (2001) reported that substorms tend to occur after a northward turning of the IMF after a southward turning. It is likely that the northward turn- ing could trigger the onset. Freeman and Morley (2009) and Newell and Liou (2011) emphasized that the initial southward turning is crucial, not just the northward turning.

\section{Geomagnetic storms}

Auroral substorms have most of the basic features of geomagnetic storms in terms of electric currents. Geomagnetic storms can be considered as a consequence of the accumulated effects of the ring current formation by auroral substorms. In fact, a substorms is a mini-storm. The period of geomagnetic storms is the period when intense substorms occur very frequently (Akasofu and Chapman, 1963), accumulating the ring current particles.

The "standard" type of geomagnetic storms consists of storm sudden commencement (ssc) and the initial, main, and recovery phases. The ssc is a step-function-like increase in the earth's magnetic field, which is followed by a fairly quiet period for a few hours of the initial phase. After the initial phase, the main phase begins, during which a large southward (reckoned negative) field develops for about 6-10h as a result of an intense ring current. The last phase is the recovery phase. Figure 10 shows an example of geomagnetic storms. It shows an assembly of low-latitude magnetic records (the $H$ component); its average gives the Dst index. Figure 10 also shows an assembly of magnetic records (the $H$ component) from high latitudes; the range between the upper and lower envelopes gives the auroral electrojet (AE) index. Except for the Chapman-Ferraro current, the main currents during geomagnetic storms are same as those of substorm currents. 


\section{Expansion phase}

(a)

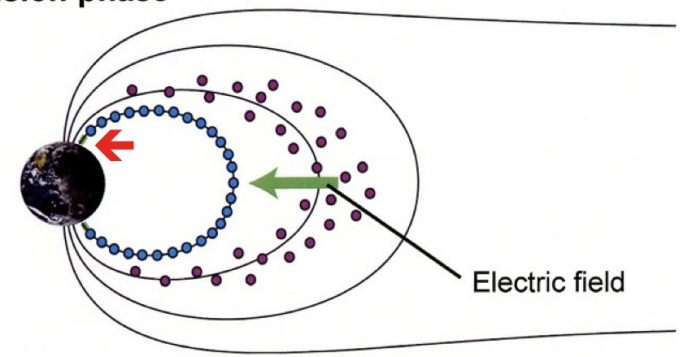

(b)
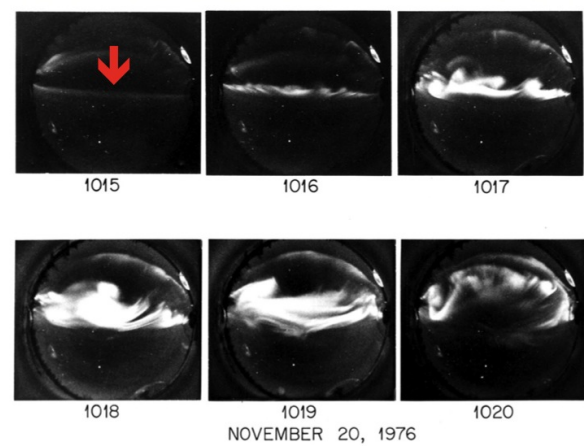

Figure 9. (a) A schematic representation of how the charge separation and the eastward electric field might occur during the deflation (Akasofu, 2017). (b) An example of a series of all-sky photographs (Fairbanks, $65^{\circ} \mathrm{gm}$ lat), showing the initial brightening (shown by a red allow) and the subsequent poleward expansion.

\subsection{Chapman-Ferraro current and ssc}

Chapman and Ferraro (1931) showed that an advancing solar plasma flow toward the earth's dipole field is stopped, because electric current is induced at the front of the plasma flow, and the Lorentz force $(J \times B)$ resulting from it is responsible for the stoppage; this current is called the Chapman-Ferraro current. Note that the magnetic field line approach cannot determine the stopping distance of the solar wind or the distance of the front side of the magnetopause. Although the advancing plasma flow is now replaced by the interplanetary shock wave, the basic process is the same. Further, since the solar wind and magnetosphere are a permanent feature of the earth, an enhancement of the plasma flow increases the Chapman-Ferraro current, reducing the stopping distance, and its effects propagate to the earth's surface, recorded as ssc.

\subsection{The ring current and the main phase}

The major difference between geomagnetic storms and auroral substorms is an anomalous development of the ring current, which is produced by a frequent occurrence (an enhanced accumulation effect) of intense substorms in about 6-12 h (Akasofu and Chapman, 1963). The intense development of the ring current causes a large depression of the horizontal component and is recognized as the main phase

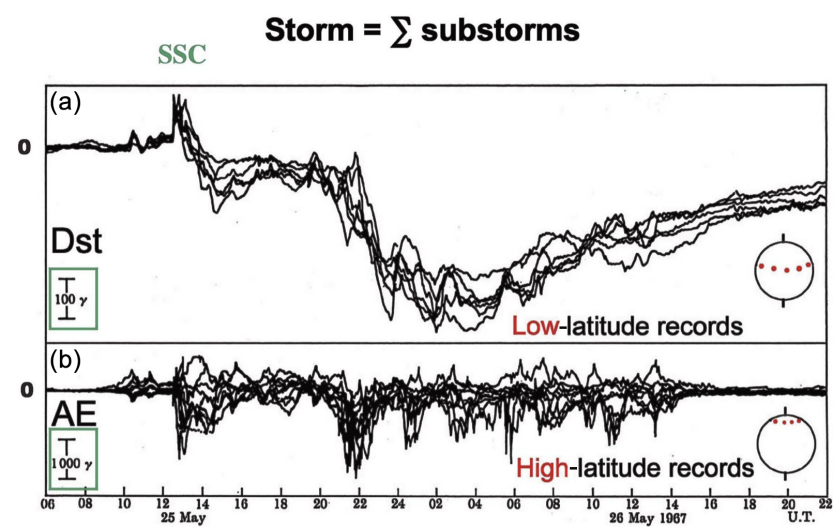

Figure 10. An example of geomagnetic storms. In the upper part, magnetic records (the $H$ component) from low-latitude stations are assembled; the Dst index is the average of the records. In the lower part, magnetic records (the $H$ component) from high latitudes are assembled; the range between the upper and lower envelopes provides the $\mathrm{AE}$ index.

of geomagnetic storms. It was found that the main ion in the ring current during intense geomagnetic storms is $\mathrm{O}^{+}$ions which are ejected from the ionosphere during intense substorms (Shelley et al., 1972; Daglis, 1977); Fig. 11a.

There has been a controversy about the relationship between substorms and storms (Sharma et al., 2003). However, as mentioned earlier, substorms are mini-storms from the point of view of the current line approach, and thus geomagnetic storms are exceptional cases during which the ring current grows anomalously large.

The current and its magnetic field are already shown in Fig. 8. The major current is the diamagnetic current that flows westward in the outer part and eastward in the inner part; the westward current has a larger effect on the earth's surface. The main phase decrease is very asymmetric during an early epoch of the development of the main phase and larger in the late evening sector (Akasofu and Chapman, 1964), indicating that the ring current particles are injected in the midnight sector and drift westward (Fok et al., 2006; Fig. 11c). The $\mathrm{O}^{+}$ions in the ring current belt become mostly neutral hydrogen atoms and can leave from the magnetosphere (the major dissipation).

\section{Heliosphere}

Although the heliosphere has become an important topic in recent years, there is so far no adequate model of the heliosphere. Most of the suggested models of the heliosphere in the past are a sort of extension of magnetospheric models based on the magnetic field line approach (cf. Balogh and Izmodenev, 2005; Longcope, 2009) and are not adequate. Thus, a little more realistic model is considered here, which is a simple model based on the principle of the unipolar induction. 

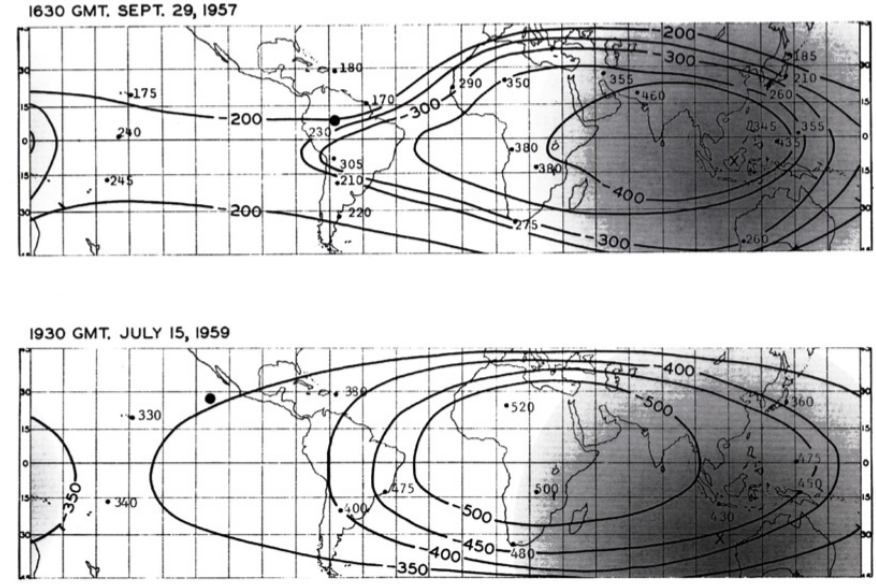

(a)
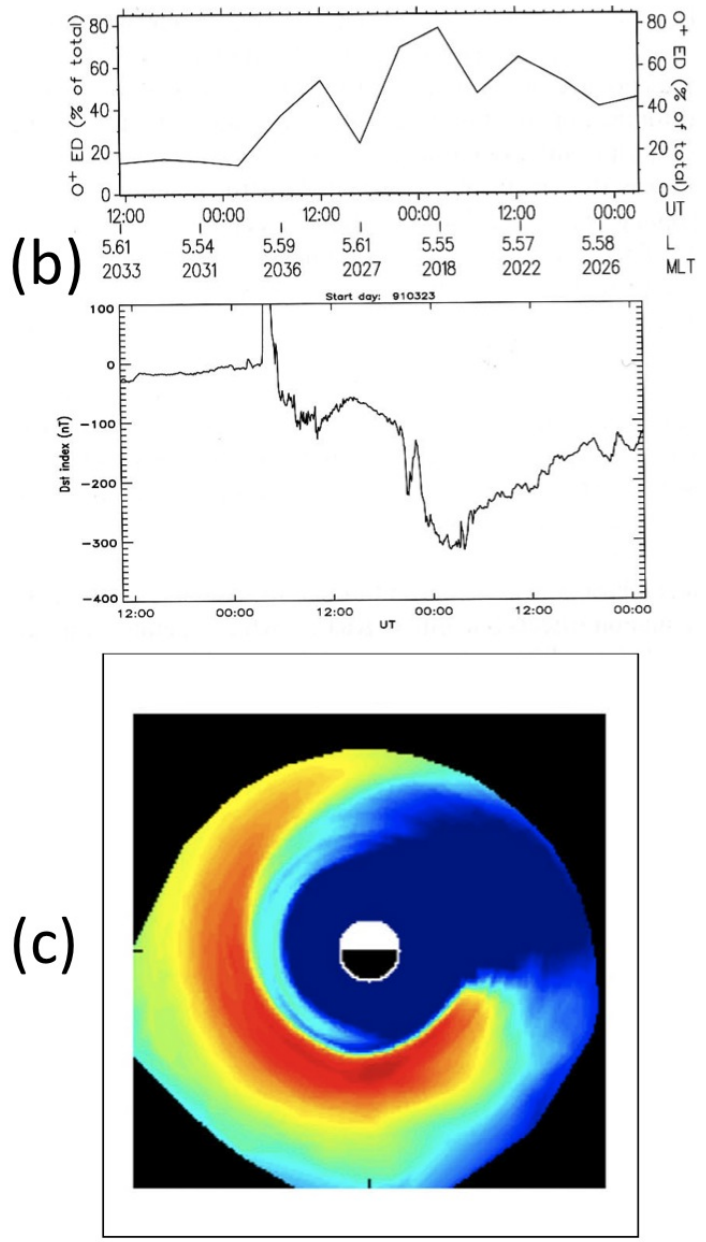

Figure 11. (a) A large asymmetric development of the main phase for two geomagnetic storms; the nightside is indicated by a dark shade (Akasofu and Chapman, 1964). (b) The $\mathrm{O}^{+}$ion flux and the Dst index (Daglis, 1977) and the simulated distribution of $\mathrm{O}^{+}$ions (Fok et al., 2006).

The main heliospheric current consists of the meridional current and the circular current in the heliospheric current sheet (HCS).

\section{Unipolar induction and the circuit}

The basic principle of modeling for the sunspot minimum period is given by Alfvén (1950). The main point of modeling is to consider the unipolar induction to drive electric currents in the heliosphere (Alfvén, 1950, Sect. 1.3; Alfvén, 1977, 279-278; 1981, chap. III). Assuming a spherical heliosphere of radius $20 R_{\mathrm{E}}$, Akasofu and Covey (1981) constructed a model in which one component of the current flows from both poles of the sun to both poles of the heliosphere. After reaching both poles of the spherical heliosphere, the two currents flow along the spherical surface and join at the equator of the heliopause and then flow radially toward the sun (radial current); the current direction reverses in each solar cycle.
There must be spiral current lines perpendicular to Parker's spiral magnetic field lines, which consist of the radial current (mentioned before) and the circular current on the HCS during the sunspot minimum period. The solar wind effect of the stretching of the field lines is represented by this circular current around the sun on the HCS, which also reverses the direction in each solar cycle. Alfvén (1981) estimated the intensity of the polar current from one hemisphere to be $1.5 \times 10^{9} \mathrm{~A}$ on the basis of the observed spiral magnetic field. Figure 12 shows two cases; in the first one, the magnetic field is confined in the heliosphere, and the second one shows the connection with an intergalactic magnetic field of intensity $0.22 \mathrm{nT}$.

In the confined case, a field line from the polar angle $\theta=10^{\circ}$, namely the latitude $80^{\circ}$, is shown as an example in both the meridional and equatorial projections in the northern hemisphere. All the field lines from lower latitudes cross the HCS after reaching the highest latitude and cross the HCS at shorter distances from the sun (Fig. 12). Although our model 

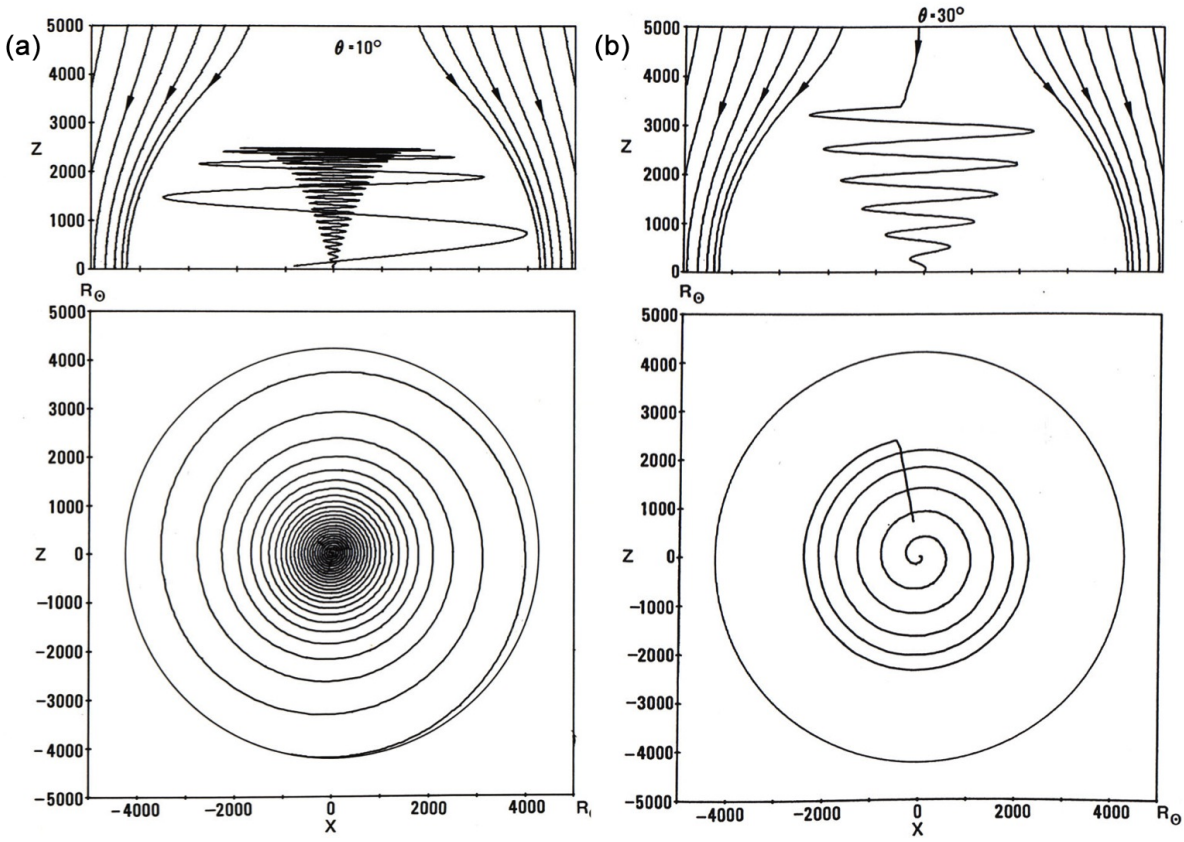

Figure 12. Two models of the heliosphere (the northern hemisphere). (a) A confined case. (b) An open case.

is very crude, the general aspect of the configuration of the field lines is expected to be similar. Such a 3-D configuration of magnetic field lines has not been shown in the past. This model satisfies both $\operatorname{div} \cdot J=0$ and $\operatorname{div} \cdot B=0$.

If the heliospheric magnetic field is not confined, highlatitude field lines of the heliosphere are expected to be connected with magnetic field lines of galactic magnetic field lines. Figure 12 shows an example of a high-latitude field line $\left(\theta=30^{\circ}\right.$; lower-latitude field lines are similar to the confined case). The model seems to reproduce the spiral structure considered by cosmic ray studies (cf. Fisk et al., 1999).

Obviously, the radius of the spherical heliosphere should be about $100 \mathrm{au}$, instead of $20 \mathrm{au}$. The motion of the solar system in the interstellar plasma can be simulated by the image dipole method (Chapman and Ferraro, 1931). In spite of this being a very primitive and crude model, it is hoped that it will be useful in studying more Ulysses observations, as well as future observations.

\section{Concluding remarks}

Most of our observed phenomena are various manifestations of electromagnetic energy dissipation, so that it is natural to study them as a chain of processes, which consists of power supply (dynamo), transmission (circuits/currents) and dissipation (flares, substorms).

In this paper, an attempt is made to study the whole solarterrestrial relationship in terms of electric currents. The electric current line approach enables us also to study not only the flow of power/energy (the basic physical quantities) from their production to the end (dissipation) in each phenomenon (solar flares, CMEs, and auroral substorms/geomagnetic storms) but also to provide the link between them in terms of their initial condition. Further, some of the crucial quantities (such as the energy consumed by a single substorm) can be obtained so far only by studying electric currents.

Electromagnetic phenomena can be studied by considering either the magnetic field line approach or the electric current line approach because of the relationship given by curl $B=J$. Although the magnetic field line approach has been almost exclusively considered in the past, it is worthwhile to take the current line approach to see if one can learn more about the processes involved. Indeed, the current line approach suggests a different way by which the power and energy are converted for our phenomena. In either case, our phenomena are quite complex and difficult. In climbing a mountain, there are at least two routes.

Data availability. The 1978 magnetograms are individually collected from the observatories listed in the paper by Kamide et al. (1982).

Competing interests. The authors declare that they have no conflict of interest.

Acknowledgements. The author would like acknowledge the late Sydney Chapman and the late Hannes Alfvén for their guidance in his early days. He would like to thank also Lou-Chuan Lee and 
Guangson Choe for their discussion on solar flares.

The topical editor, Georgios Balasis, thanks two anonymous referees for help in evaluating this paper.

\section{References}

Akasofu, S.-I.: Physics of Magnetospheric Substorms, 599 pp., D. Reidel Pub. Cp., Dordrecht-Holland, 1977.

Akasofu, S.-I.: Where is the magnetic energy for the expansion phase of auroral substorms accumulated?, J. Geophys. Res., 118, 7219-7225, https://doi.org/10.1002/2013JA019042, 2013.

Akasofu, S.-I.: Auroral substorms as an electrical discharge phenomenon, Progress in Earth and Planetary Science, 2, 20, https://doi.org/10.1186/s40645-015-0050-9, 2015.

Akasofu, S.-I.: Auroral Substorms: Search for Processes Causing the Expansion Phase in Terms of the Electric Current Approach, Space Sci. Rev., 1-41, https://doi.org/10.1007/s11214017-0363-7, 2017.

Akasofu, S.-I. and Chapman, S.: The ring current, Geomagnetic disturbances and the Van Allen radiation belts, J. Geophys. Res., 66, 1321-1350, 1961.

Akasofu, S.-I. and Chapman, S.: Magnetic storms: The simultaneous development of the main phase (DR) and polar magnetic substorms (DP), J. Geophys. Res., 68, 155-158, 1963.

Akasofu, S. and Chapman, S.: On the asymmetric development of magnetic storm fields in low and middle latitudes, Planet. Space Sci., 12, 905-12913, 1964.

Akasofu, S.-I. and Covey, D. N.: Magnetic field configuration of the helosphere in interstellar space, Planet. Space Sci., 29, 313-316, 1981.

Akasofu, S.-I., Cain, J. C., and Chapman, S.: The magnetic field of a model radiation belt, numerically computed, J. Geophys. Res., 66, 4013-4026, 1961.

Alfvén, H.: Cosmical Electrodynamics, Oxford Univ. Press, Oxford, UK, 1950.

Alfvén, H.: The second approach to cosmical electrodynamics, in: The Birkeland Symposium on aurora and magnetic storms, 439444, edited by: Egeland, A. and Holtet, J., Centre Natinal de la Recherche Scientifique, 1967.

Alfvén, H.: Electric currents in cosmic plasmas, Rev. Geophys.Space, 15, 272-284, 1977

Alfvén, H.: Cosmic Plasma, 164 pp., D. Reidel Pub. Co., DordrechtHolland, 1981.

Alfvén, H. and Carlqvist, P.: Current in the solar atmosphere and a theory of solar flares, Solar Phys., 1, 220-228, 1967.

Aschwanden, M.: Physics of the Solar Corona: An Introduction, 842 pp., Springer, 2004.

Aschwanden, M. J.: Magnetic energy dissipation during the 2014 March 29 solar flare, Astrophys. J. Lett., 804, 6 pp., 2015.

Aschwande, M. J., Boerner, P., Ryan, D., Caspi, A., NcTiernan, J. M., and Warren, H. P.: Global energetics of solar flares, I. Magnetic energies, Astrophys. J., 797, 20 pp., https://doi.org/10.1088/0004.-637X/797/1/50, 2014.

Aschwanden, M. J., Xu, Y., and Jing, J.: Global energetics of solar flares, II Thermal energies, Astrophys. J., 802, 53, https://doi.org/10.1088/0004-637X/802/1/53, 2015.
Axford, W. I. and Hines, C. O.: A unified theory of high latitude geophysical phenomena and geomagnetic storms, Can. J. Phys., 39, 1433-1464, 1961.

Balogh, A. and Izmodenov, V.: The heliosphere and its boundary, in: The solar system and Beyond: Ten years of ISSI, edited by: Geiss, J. and Hultqvist, B., 151-163, International Space Science Institute, 2005.

Bostrom, R.: A model of the auroral electrojets, J. Geophys. Res., 69, 4983-4000, 1964.

Bothmer, V. and Schwenn, R.: Eruptive prominences of magnetic clouds in the solar wind, Space Sci. Rev., 70, 215-220, 1994.

Burlaga, L., Sitter, E., Marini, F., and Schwenn, R.: Magnetic loop behind a interplanetary shock: Voygr, Helios and IMP 8 observations, J. Geophys. Res., 86, 6673-6684, 1981.

Chapman, S.: The electric current-systems of magnetic storms, Terr. Mag., 40, 349-370, 1935.

Chapman, S. and Ferraro, V. C. A.: A new theory of magnetic storms, I. Initial phase, Terr. Mag., 36, 77-97, 1931.

Chen, J. and Krall, J.: Acceleration of coronal mass ejection, J. Geophys. Res., 108, 1410, https://doi.org/10.1029/2003JA009849, 2003.

Choe, G. S. and Lee, L. C.: Evolution of solar magnetic arches, I. Ideal MHD evolution under footpoint shearing, Astrophys. J., 472, 360-388, 1996a.

Choe, G. S. and Lee, L. C.: Evolution of magnetic arcades, II. Effects of resistivity and solareruptive processes, Astrophys. J., 472, 372-388, $1996 \mathrm{~b}$.

Daglis, I. A.: Mission study oxygen issue, EOS, 78, 245-251, 1977.

Dungey, J. W.: Interplanetary magnetic field and the auroral zones, Phys. Rev. Lett., 6, 47-48, 1961.

Fisher, G. H., Abb, W. P., Bercik, Kaza, M. D., Lynch, B. J., Welsh, B. T., Hoeksema, J. T., Hayashi, K., Liu, Y., Nortomn, A. A. , Dalda, A. S., Sun, X., DeRosa, M. L., and Cheung, M. C. M.: The coronal global evolutionary model: Using HMI vector magnetogram and Doppler data to model the buildup of free magnetic energy in the solar corona, Space Weather, 13, 369-373, https://doi.org/10.02/2015SW001191, 2015.

Fisk, L. A., Zurbuchen, T. H., and Schwadron, N. A.: On the coronal magnetic field: Consequences of large-scale motions, Astrophys. J., 521, 868-877, 1999.

Fletcher, L. and Hudson, H. S.: Impulsive phase flare energy transport by large-scale Alfvén waves and electron acceleration problem, Astrophys. J., 675, 1645-1655, 2008.

Fok, Mc., Mre, T. E., Brandt, P. C., Delcourt, D. C., Slinker, S. P., and Fedder, J. A.: Impulsive enhancement of oxygen ions during substorms, J. Geophys. Res., 111, A10222, https://doi.org/10.1029/2006JA011839, 2006.

Freeman, M. P. and Morley, S. K.: No evidence for externally triggered substorms based on superposed epoch analysis of IMF Bz, Geophys. Res. Lett., 26, L21101, https://doi.org/10.1029/2009GL040621, 2009.

Fry, C. D., Sun, W., Deehr, C. S., Dryer, M., Smith, Z., Tokumaro, M., Kojima, M., and Akasofu, S.-I.: Improvement to the HAF solar wind model for space weather prediction, J. Geophys. Res., 106, 20985-21001, 2001.

Filippov, B. and Koutchmy, S.: Causal relationships between eruptive prominences and coronal mass ejections, Ann. Geophys., 26, 3025-3031, https://doi.org/10.5194/angeo-26-3025-2008, 2008. 
Ge, Y. S. and Russell, C. T.: Polar survey of magnetic field in neartail: Reconnection rare inside 9 Re, Geophys. Res. Lett., 33, LO2101, https://doi.org/10.1029/2005GL024574, 2006.

Gosling, J. T., Baker, D. N., Bame, S. J., and Zwickl, R. D.: Bidirectional solar wind electron flux and hemispherically symmetric polar rain, J. Geophys. Res., 91, 11352-11358, 1986.

Hirayama, T.: Theoretical model of flares and prominences: 1. Evaporation flare model, Solar. Phys., 34, 323-338, 1974.

Hudson, H. S.: Global propertices of solar flares, Space Sci. Rev., 158, 5-41, https://doi.org/10.1007/s11214-010972-4, 2011.

Kamide, Y., Ahn, B.-H., Akasofu, S.-I., Baumjohhann, W., FriisChristensen, E., Kroehl, H., Mauer, A., Richmond, A. D., Rostoker, G., Spiro, R. W., Walker, J., and Zeizev, A.: Global distribution of ionospheric and field-aligned currents as determined from six IMS meridian chains of magnetometers: Initial results, J. Geophys. Res., 87, 8228-8240, 1982.

Kazachenko, M. D., Fisher, G. H., Weksch, B. T., Liu, Y., and Sun, X.: Photospheric electric fields and energy fluxes in the eruptive active region NOAA 11158, Astrophys. J., 811, 16, https://doi.org/10.1088/0004-637X/811/1/16, 2015.

Kerr, G. S. and Fletcher, L.: Physical propertices of white-light sources in the 2011 Feb.15 solar flare, Astrophys. J., 20, 1-15, 2014.

Kosovichev, A. G. and Duvall Jr., T. L.: Investigation of a sunspot complex by time-distance helioseismology, The Physics of the Sun and Star Spots, Proceedings IAU Symposium, No. 273, edited by: Chodhary, P. D. and Strassmeter, K. G., 2010.

Kurokawa, H., Hanaoka, Y., Shibata, K., and Uchida, Y.: Rorating eruption of untwisting filament triggered by the 3B flare of 25 April, Solar Phys., 108, 251-264, 1987.

Longcope, D. W.: Magnetic field topology, in: Heliospheric Physics, edited by: Schrijve, C. and Sisco, G. L., 77-112, Cambridge Univ. Press, 2009.

Lugaz, N., Vourlidas, A., Roussev, I. I., and Morgan, H.: Solar-Terrestrial simulationin the STEREO Era: The 2425 January 2007 eruption, Solar Phys., 256, 269-284, https://doi.org/10.1007/s11207-009-9339-4., 2009.

Lui, A. T. Y. and Kamide, Y.: A fresh perspective of the substorm current system and its dynamics, Geophys. Res. Lett., 30, 1958, https://doi.org/10.1029/2003GL017835, 2003.

Milligan, R., McAteer, R. T. J., Dennis, B. R., and Young, C. A.: Evidence of a plasmoid-looptop interaction and magnetic inflows during a solar flare/coronal mass ejection eruptive event, Astrophys. J., 713, 1292-1300, https://doi.org/10.1088/0004637X/713/2/1292, 2010.

Moore, R. L., Sterling, A. C., Hudson, H., and Lemen, J. R.: Onset of the magnetic explosion in solar flares and coronal mass ejections, Astrophys. J., 552, 833-848, 2001.

Newell, P. T. and Liou, K.: Solar wind driving and substorm triggering, J. Geophys. Res., 116, A03229, https://doi.org/10.1029/2010JA016139, 2011.

Odstrcil, D., Riley, P., and Zao, X. P.: Numerical simulation of the 12 May 1997 interplanetary CME event, J. Geophys. Res., 109, A02116, https://doi.org/10.1029/2003JA010135, 2004.

Rust, D. M. and LaBonte, B. J.: Observational evidence of the kink instability in solar filament eruptions and sigmoids, Astrophys., 622, L69-L72, 2005.

Saito, T., Sun, W., Deehr, C. S., and Akasofu, S.-I.: Transequatorial magnetic flux loops on the sun as a possible new source of geomagnetic storms, J. Geophys. Res., 112, A05102, https://doi.org/10.1029/2006JA01194, 2007.

Schuck, P. W.: The photospheric energy and helicity budgets of the flux-injection hypothesis, Astrophys. J., 714, 68-88, https://doi.org/10.1088/0004-637X/714/1/68, 2010.

Sharma, A. S., Kamide, Y., and Lakhina, G. S.: Disturbances in Geospace: The storm-substorm relationship, AGU Monograph 142, AGU, Whasington DC, 2003.

Shelley, E. G., Johson, R. G., and Sharp, R. D.: Satellite observations of energetic heavy ions during geomagnetic storm, J. Geophys. Res., 77, 6104-6110, 1972.

Shibata, K. and Magara, T.: Solar flares: Magnetohydrodynamic processes, Living Rev. Solar Phys., 8, 6 pp., 2011.

Steward, G. A., Lobzin, V. V., Wilkinson, P. J., Cairns, I. V., and Robinson, P. A.: Automatic recognition of complex magnetic regions on the sun in GONG magnetogram images and prediction of flares: Techniques for the flare warning program flarecast, Space Weather, 9, S11004, https://doi.org/10.1029/2011SW000703, 2011.

Su, Y., Veronig, A. M., Holman, G. D., Dennis, B. R., Wang, T., Temmerand, M., and Gan, W.: Imaging coronal magnetic-field reconnection in a solar flare, Nat. Phys., https://doi.org/10.1038/NPHY52675, 2013.

Sun, W., Xu, S.-I., and Akasofu, S.-I.: Mathematical separation of directly-driven and unloading components in the ionospheric equivalent currents during substorms, J. Geophys. Res., 103, 11695-1170, 1998.

Svestka, Z.: Solar Flares, D. Reidel Pub. Co., Dordrecht-Holland, 399 pp., 1976.

Sweet, P. A.: The neutral point theory of solar flares, in: Electromagnetic Phenomena Cosmical Physics, edited by: Lehnert, B., 123-134, Cambridge Univ. Press, 1958.

Wang, H. and Liu, C.: Observational evidence of back reaction on the solar surface associated with coronal magnetic restructuring in solar eruptions, Astrophys. J., 716, 195-199, https://doi.org/10.1088/2041-8205/716/2/1.195, 2010.

Webb, D. F., Cliver, E. W., Crooker, N. U., St. Cyr, O. C., and Thompson, B. J.: Relationship of halo coronal mass elections, maggetic cloud, and magnetic storms, J. Geophys. Res., 105, 7491, 2000.

Wu, C. C., Fryer, C. D., Wu, S. T., Dryer, M., and Liou, K.: Three-dimentional global simulation of interplanetary coronal mass ejection propagation from the sun to the heliosphere: Solar event of 12 May 1997, J. Geophys. Res., 112, A09104, https://doi.org/10.1029/2006JA012211, 2007.

Zao, N. M.: Observations of photospheric dynamics and magnetic fields: From large-scale to small-scale flows, Space Sci. Rev., 144, 127-149, https://doi.org/10.1007/s11214.088.0472.7, 2009.

Zharkova, V. V.,Arzner, K., Benz, A. O., Browning, Dauphin, C., Emslie, A. G. Flecher, L., Kontar, E. P., Mann, G., Onofri, M., Petrosian, V., Turkmani, Vilmer, N., and Vlahos, L.: Recent advances in understanding particle acceleration processes in solar flares, Space Sci. Rev., 159, 357-420, https://doi.org/10.1007/s11214-011-9803-y, 2011.

Zhou, X.-Y. and Tsurutani, B. T.: Interplanetary shock effects on the nightside auroral zone, magnetosphere and ionosphere, in: Space Weather Study Using Multipoint Techniques, edited by: Lyu, L.H., 139-147, Pergamon, 2002. 